\title{
Can an integrated sexual health service function effectively without a health advisor?
}

\section{Jennifer McCay, Specialist Sexual Health Nurse Trafford Sexual Health Services}

\author{
Bridgewater Community Healthcare WHS
}

Background Four traditional genitourinary medicine (GUM) and family planning services merged to create one integrated sexual health service in Trafford in 2013. This service currently operates without a health advisor. Basic health advising duties, including partner notification (PN) are carried out by specialist nurses.

Objective To audit the effectiveness of current practice against the standards of the BASHH partner notification guidelines for chlamydia ${ }^{1,2}$ and to consider changing practice and/or service provision if adherence was found to be poor.

Method Patients with a diagnosis of chlamydia that attended the service between 01/08/15 - 30/09/15 were included. The notes were reviewed retrospectively and audited against the standards of the BASHH partner notification guidelines ${ }^{1,2}$.

\section{Results}

90 patients were identified with chlamydia during the audit period. $96 \%$ of patients had PN discussed with them at the time of treatment. 57\% had PN agreed for each contact and PN outcomes were documented.



0.66 contacts per index patient were reported as having attended. 0.3 contacts per index patient were verified as having attended.

\section{BASHH Guideline}



\section{Audit Finding}

Reported



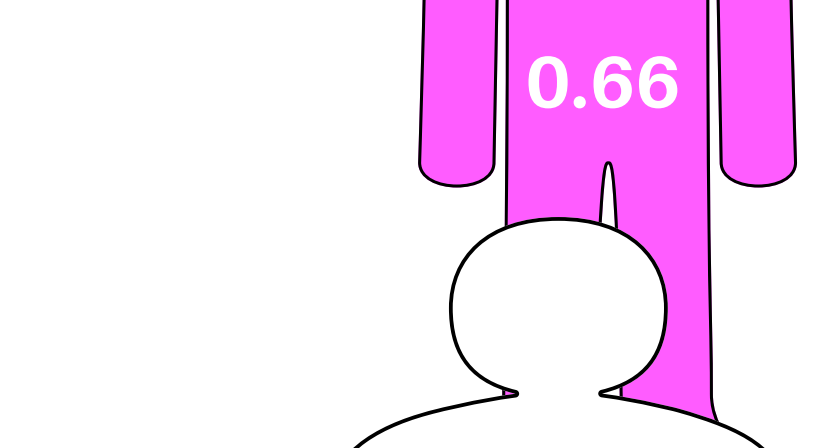
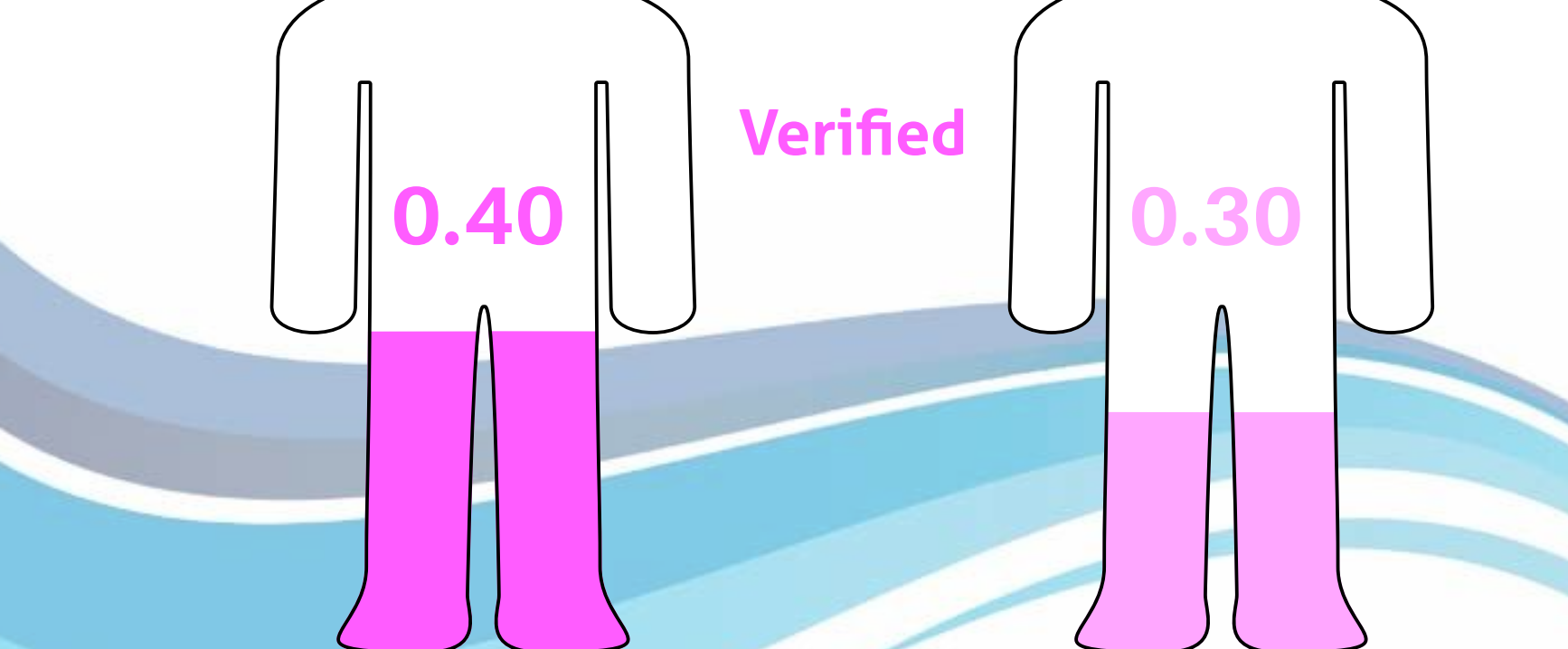

$79 \%$ of patients had a follow-up telephone call/ consultation. Of these, $98 \%$ had a compliance check documented and $42 \%$ had an abstinence check documented.

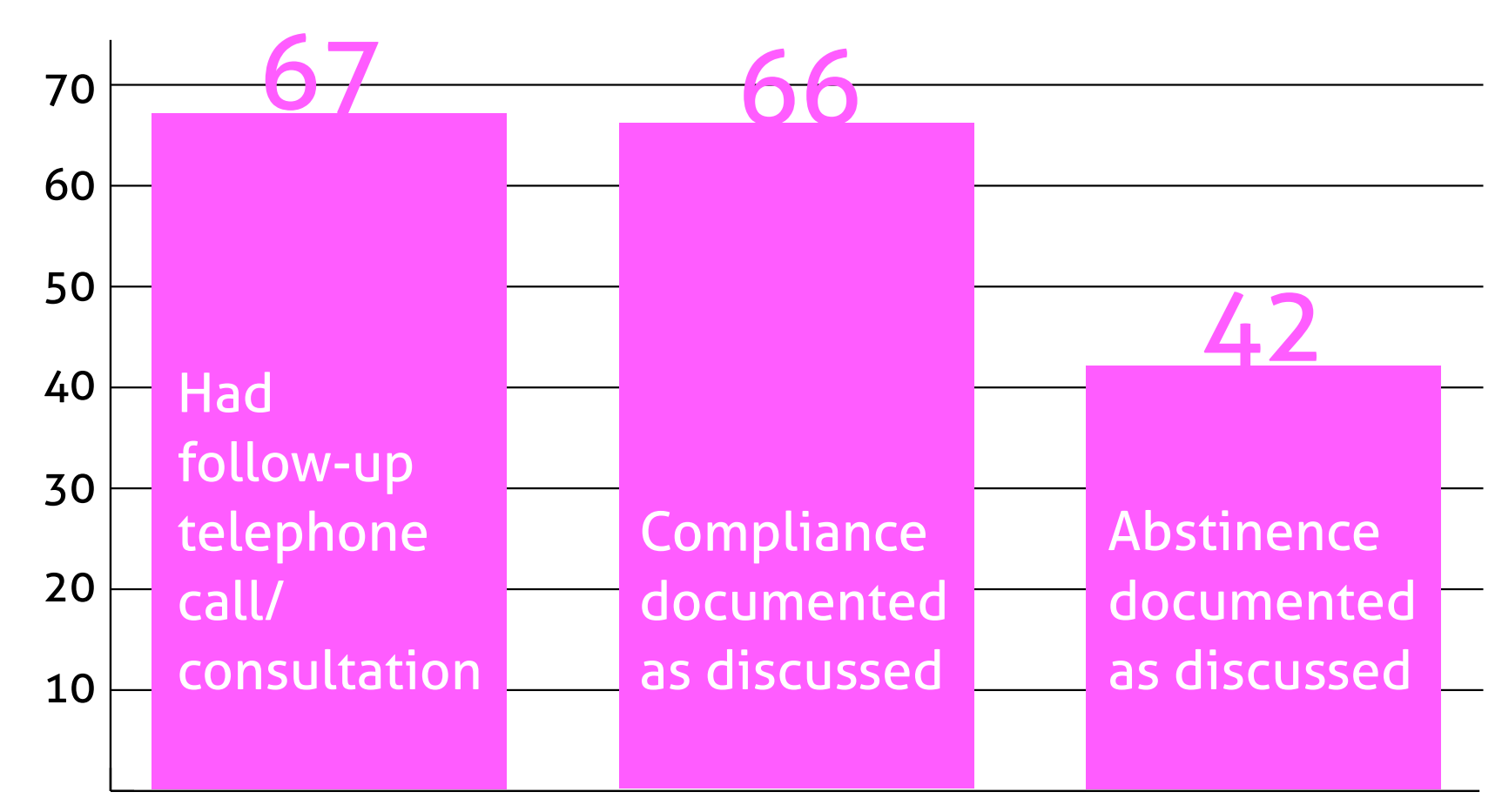

\section{Conclusion}

Adherence to BASHH PN standards was better than expected but verified PN still falls below the guidelines. Measures have been taken to improve adherence. These include:

- Prompts on the new Electronic Patient Records system to initiate and review PN

- The local recalls policy has been updated

- A compliance check proforma has been introduced.

The audit demonstrates the need for a health advisor to be responsible for PN within an integrated service to improve adherence to national standards for PN.

Recruitment of a health advisor for the service was discussed and agreed as a result of this audit, but is subject to the outcome of a current tendering process.

References: ${ }^{1} \mathrm{BASHH}$ (2012) BASHH Statement on Partner Notification for Sexually Transmissible Infections

2BASHH (2006) UK National Guideline for the Management of Gentital Tract Infection with Chlamydia Trachomatis

Thanks go to Bridgewater NHS Foundation Trust Audit Team. 